\title{
Impact of Left Bundle Branch Block on Left Ventricular Mechanics in Patients with Idiopathic Dilated Cardiomyopathy
}

\author{
Ahmed Emara1, Hala Mahfouz Badran1,2, Waleed Abdou1, Naglaa Fahim1, Mohamed Fathi', \\ Magdi H. Yacoub2,3 \\ ${ }^{1}$ Cardiology Department, Menoufiya University, Menoufia Governorate, Egypt \\ ${ }^{2}$ The BAHCM National Program, Menoufia Governorate, Egypt \\ ${ }^{3}$ Imperial College, London, UK \\ Email: am.emara@yahoo.com
}

How to cite this paper: Emara, A., Badran, H.M., Abdou, W., Fahim, N., Fathi, M. and Yacoub, M.H. (2019) Impact of Left Bundle Branch Block on Left Ventricular Mechanics in Patients with Idiopathic Dilated Cardiomyopathy. World Journal of Cardiovascular Diseases, 9, 132-148. https://doi.org/10.4236/wjcd.2019.92013

Received: January 5, 2019

Accepted: February 23, 2019

Published: February 26, 2019

Copyright $\odot 2019$ by author(s) and Scientific Research Publishing Inc. This work is licensed under the Creative Commons Attribution International License (CC BY 4.0).

http://creativecommons.org/licenses/by/4.0/

\begin{abstract}
Objectives: Left bundle branch block (LBBB) is commonly associated with heart failure. We evaluated the prevalence and impact of LBBB on left ventricular mechanics using 2D strain imaging in patients with idiopathic dilated cardiomyopathy (IDCM). Methods: We included 101 patients with IDCM with mean age $38 \pm 18$ years: $74 \%$ were males and $13.9 \%$ of them were in NYHA Class III-IV. LBBB was present in 26 (37\%) of included patients. Myocardial mechanics including longitudinal, circumferential strain and rotation and LV synchronization were assessed using two-dimensional strain imaging. Results: LBBB group had higher LV volumes, and PAP compared with non LBBB. Peak LV longitudinal systolic strain $\left(\varepsilon_{\text {sys }}\right)$ of the septum and global $\mathrm{LV} \mathrm{SR}_{\text {sys }}$ were significantly lower in $\mathrm{LBBB}$ compared to non $\mathrm{LBBB}$ group ( $\mathrm{P}<0.01,<0.03)$. TTP-d was greater in LBBB in comparison to non LBBB group $(274.5 \pm 116$ versus $209.4 \pm 139, \mathrm{P}<0.02)$. The electromechanical delay between septal segments was $35 \pm 18 \mathrm{~ms}$ and between lateral wall segments: $48 \pm 24 \mathrm{~ms}$, between anterior wall segments: $21 \pm 11$ and between inferior wall segments: $41 \pm 12$. Consequently, LV mechanical dyssynchrony was more evident in IDCM patients with LBBB. QRS width was correlated inversely with LV longitudinal strain and strain rate and electromechanical delay $(\mathrm{P}<0.0001)$ in non LBBB group. In LBBB QRS width was not related to cardiac mechanics. Using univariate analysis and after a multiple covariate adjustment, the baseline LBBB was associated with a significantly increased LV dysfunction. Conclusion: After correcting for potential confounders, LBBB was found to be associated with more deterioration of LV mechanics and exaggerated LV dyssynchrony in patients with IDCM.
\end{abstract}




\section{Keywords}

Idiopathic Dilated Cardiomyopathy, LBBB, 2D Strain Imaging

\section{Introduction}

Idiopathic dilated cardiomyopathy (IDCM) is a diagnosis that continues to puzzle many cardiovascular specialists. A number of Left bundle branch block (LBBB) occurs frequently in patients with dilated cardiomyopathy (DCM) and is associated with high cardiac morbidity and mortality. The characteristic feature of LBBB is an asynchronous septal wall motion and most frequently a delay of the lateral and/or posterior wall segments [1]; this heterogeneous activation of the ventricle leads to inefficient contraction.

Experimental data suggest that the redistribution of local workload induced by LBBB provokes substantial changes in regional myocardial blood flow and glucose metabolism along with structural remodeling [2]. It is generally assumed that LBBB occurs secondarily to the underlying disease process of DCM; however, the possibility exists that in some patients the LBBB-induced abnormal left ventricular (LV) contraction pattern could-over long term-induce DCM [3].

DCM patients with LBBB, as compared to those with normal intraventricular conduction, are more likely to have profound LV dilatation, lower ejection fraction, increased symptomatology, and shorter survival. There is extensive research underway in patients with DCM and LBBB to evaluate the short and long-term effects of normalization of ventricular activation sequence with high septal, LV, or biventricular pacing [4].

Recent studies showed that LV deformation in the longitudinal plane estimated by speckle tracking is significantly reduced in patients with DCM, when compared with normal individuals [5]. The objective of this study was to investigate the impact of LBBB on LV deformation as measured by two-dimensional strain imaging in patients with IDCM.

\section{Patients and Methods}

\subsection{Study Population}

\section{1) IDC Group}

The patients participating in this prospective study were selected from a series of consecutive patients referred to our center (Yacoub Research Unite, Menoufia University, Egypt) between March 2013 and January 2015. IDCM was diagnosed on the basis of patients' clinical history, physical examination, 12-lead ECG, chest radiography, echocardiography, and coronary angiography (for patients > 40 years old) according to the World Health Organization criteria [6]. Echocardiographic diagnosis of IDCM was based on the following criteria: the presence of an ejection fraction of $<0.45$ or fractional shortening of $<0.25$ and a left ventricular end-diastolic diameter $>117 \%$ of that expected for the patient's 
age and body surface area [7]. 101 IDCM patients (mean age $32.5 \pm 15$ ) with and without LBBB were enrolled in the study after their informed consent, and approval of Ethics Committee of Menoufia University Hospitals was obtained.

Patients were excluded from the study if LV dysfunction was secondary to one of the following: hypertension $(0.160 / 100 \mathrm{mmHg})$, significant coronary artery disease history of, tachycardia-induced cardiomyopathy, chronic dyssrhythmias like atrial fibrillation, Cor pulmonale, diseases of pericardium, or congenital heart diseases.

At the time of enrolment, all patients underwent a complete clinical evaluation, which included an accurate clinical history and a physical examination, 12-lead electrocardiogram (ECG), standard X-ray, M-Mode, two-dimensional, Doppler echocardiography, and speckle tracking echocardiography.

ECG measurements were assessed from a standard 12-lead ECG. Complete LBBB was defined as: a notching or slurring QRS longer than 120 milliseconds with an initial $\mathrm{R}$ wave in lead I and aVL, the left precordial leads and displacement of the S-T segment, and usually the $\mathrm{T}$ wave, in a direction opposite to that of the principal QRS deflection, absent q waves; wide, slurred R waves in V5 and V6; and monophasic QS or rS waves in V1 and V2 [8].

All patients were allowed to receive their medications including angiotensin converting enzyme (ACE) inhibitors and diuretics. Beta-blockers were added at the highest tolerated dosage. Digoxin was associated when considered necessary.

\section{2) Conventional Echocardiography}

Echocardiographic images were obtained in the parasternal long, short-axis, apical 2 and 4-chamber views using standard transducer positions. Esaote Mylab Gold ultrasound system (Esaote S.p.A, Florence, Italy) equipped with a $5 \mathrm{MHz}$ phased-array transducer was utilized. LV end diastolic (LVDd), end systolic diameter (LVSd), septum (SPT), posterior wall thickness (PWT), Ejection Fraction (EF\%) and left atrial (LA) diameter \& volume were measured in accordance with the recommendations of the American Society of Echocardiography [9]. Continuous-wave Doppler was used to estimate pulmonary artery pressure from tricuspid regurg velocity (Bernolli equation). Severity of MR was graded according to the jet area method [10]. Peak early (E) and late (A) transmitral filling velocities were measured from mitral inflow velocities.

\section{3) Analysis of LV Deformation}

LV images were recorded and processed. Tracking and subsequent strain calculations were performed with the software package Esaote-X-Strain based on a previously validated algorithm [11]. Scanning was performed longitudinally from the apex to acquire best apical views. Frame rate $(70 \pm 20 \mathrm{~F} / \mathrm{s})$, was adjusted depending on the heart rate. $\varepsilon_{\mathrm{sys}}, \mathrm{SR}_{\mathrm{sys}}, \mathrm{SR}_{\mathrm{e}}$ and $\mathrm{SR}_{\mathrm{a}}$ in the basal, mid and apical segments of septal, lateral, anterior and inferior wall were measured. In order to reduce random noise, each sample was obtained by averaging more than one consecutive heart cycle (usually three) (Figure 1). Peak early diastolic $\left(E_{a}\right)$ velocity was obtained by placing a tissue Doppler (TDI) sample volume at the septal 

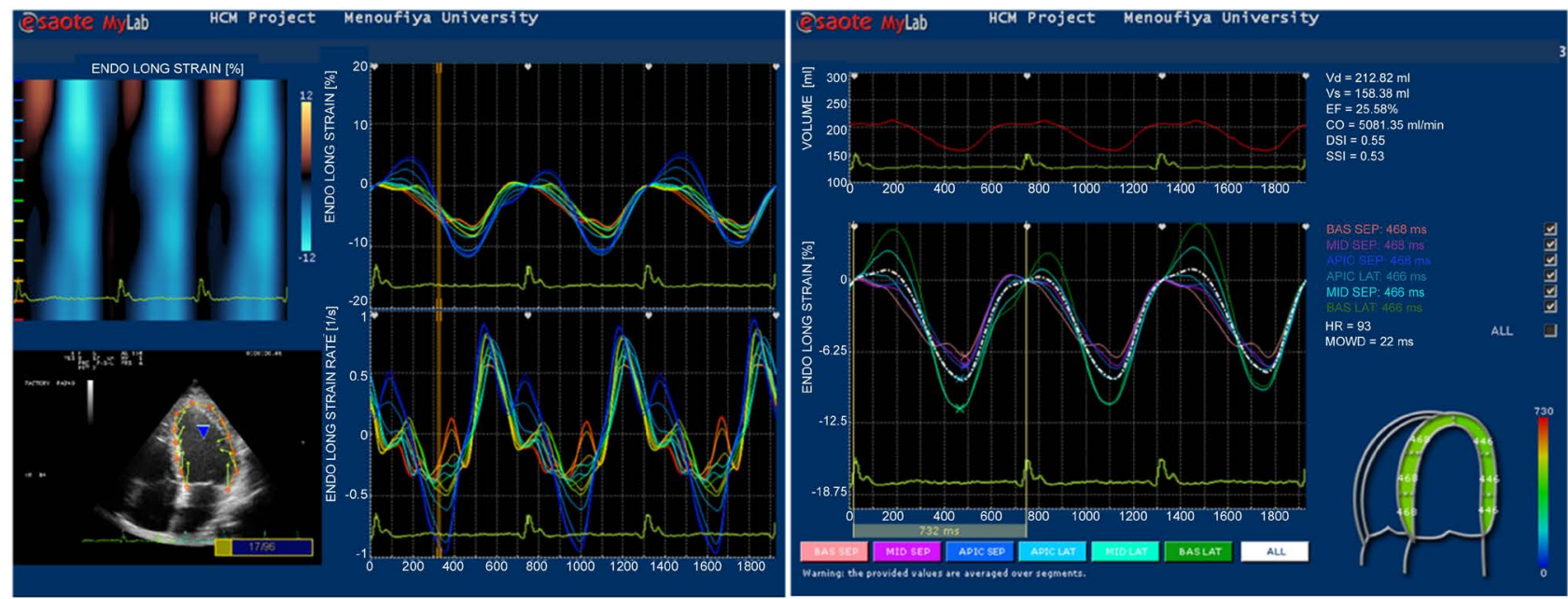

Figure 1. Peak systolic strain and strain rate of LV segments in apical $4 \mathrm{CH}$ view in a patient with IDCM and non LBBB. Global longitudinal strain is $6.5 \%$ and electromechanical delay was $22 \mathrm{~ms}$.

and lateral mitral annulus in the apical 4-chamber view and the mean value was obtained. The $\mathrm{E} / \mathrm{E}_{\mathrm{a}}$ ratio was also calculated.

Circumferential strain and LV twist and untwist rate were assessed from short-axis views at the basal and apical levels. Care was taken to ensure that the basal short-axis plane contained the mitral valve and that the apical plane was acquired distally to the papillary muscle with the left ventricle as circular as possible and proximal to the level with luminal obliteration at end-systole [12]. LV twist was calculated as the instantaneous difference between apical and basal rotation [13].

To estimate LV mechanical dyssynchrony, myocardial contraction time was measured from regional strain curves for each ventricular segment, as time from the beginning of Q wave of ECG to the time to peak systolic strain (TTP). Intraventricular electromechanical delay (EMD) was measured as the difference of time to peak systolic strain (d-TTP) in eighteen LV myocardial segments obtained from apical views (difference between the longest and shortest cycle) [14] [15]. LV dyssynchrony was defined as the standard deviation of the averaged time-to-peak-strain (TTP-SD).

\subsection{Statistical Analysis}

Variables are presented as numbers and percentages or mean $+\mathrm{SD}$, as indicated. The distribution of qualitative variables among patient groups was analyzed by Chi-square test or Fisher's exact test, as appropriate. Means were compared with the unpaired Student's test. QRS duration was correlated with other variables using the correlation coefficient $r$ in all DCM patients, as well in both subgroups: with and without LBBB. ROC analysis was used to discriminate between patients with and those without LBBB and to calculate the corresponding sensitivity and specificity of significant variables. All tests were bilateral and a value of 0.05 or less was our limit for statistical significance. All calculations were made b IBM SPSS version 23 for MAC (11). 


\section{Results}

\subsection{Clinical Characteristics}

A total of 101 patients with IDCM were enrolled. All patients were in sinus rhythm during the acquisition of echocardiography. The mean (SD) age was 38 \pm 18 years; $74 \%$ were males and $13.9 \%$ of them were in NYHA Class III-IV. LBBB was present in 26 (37\%) of included patients.

Accordingly, patients were categorized into two groups. LBBB Group consisted of 26 IDCM patients with LBBB and Non LBBB Group consisted of 75 IDCM patients without LBBB.

From ECG data, QRS duration was significantly prolonged in LBBB compared to non LBBB group $137 \pm 21$ versus $60 \pm 20 \mathrm{~ms}, \mathrm{P}<0.0001$. In non LBBB group there was 15 patients $(20 \%)$ had QRS duration $\geq 120$ as intraventricular conduction delay (IVCD).

Demographic and clinical characteristics of studied groups are outlined in Table 1, LBBB group showed higher prevalence of female compared to non LBBB group $(P=0.03)$. No differences between LBBB group and non-LBBB group in body surface area (BSA), heart rate, systolic or diastolic blood pressure. No difference between groups in familial incidence, New York Heart Association (NYHA) functional class, $(\mathrm{P}=\mathrm{NS})$.

\subsection{Conventional Echocardiographic Data}

From all conventional echocardiographic variable In LBBB group LV EDD and ESD were significantly greater compared to non LBBB group $(\mathrm{P}=0.02,0.03$ respectively). Also LBBB group had higher pulmonary artery pressure (PAP) compared to non LBBB group $(\mathrm{P}=0.03)$. Despite the trend for a difference in left ventricular mass index (LVMI) $(\mathrm{P}=0.08)$ it did not reach statistical significance. Nevertheless, no important difference in severity of mitral regurgitation was detected between the two subgroups. No relevant difference was noticed between patients with LBBB group versus the non-LBBB group in LA size, LV EF\% and all other conventional echocardiographic variables. The pharmacological treatment did not differ significantly between the groups.

\subsection{Deformation Analysis}

Global longitudinal $\varepsilon_{\text {sys }}, \mathrm{SR}_{\mathrm{sys}}, \mathrm{SR}_{\mathrm{e}}$ and $\mathrm{SR}_{\mathrm{a}}$ were calculated from $12 \mathrm{LV}$ segments (Tables 2-4, Figure $1 \&$ Figure 2). The value of the peak LV longitudinal systolic strain $\left(\varepsilon_{\text {sys }}\right)$ of the septal segments were significantly lower in LBBB compared to non LBBB group at segmental level and at whole septal wall $(\mathrm{P}<0.01)$. Similarly $\varepsilon_{\text {sys }}$ of lateral and anterior wall segments showed marked reduction of strain values $(\mathrm{P}<0.02)$. Despite the impaired longitudinal strain in all IDCM patients, those with LBBB showed marked deterioration compared to non-LBBB group at the septum $(-3.12 \pm 2.92$ versus $-5.51 \pm 4.45, \mathrm{P}<0.005)$ and at global level $(-3.17 \pm 2.48$ and $-5.27 \pm 3.84$ respectively $)(\mathrm{P}<0.01)$.

Similarly, LV SR $\mathrm{sys}_{\text {s }}$ showed reduction of septal segments in LBBB compared to 
Table 1. Clinical and conventional echocardiogaphic data.

\begin{tabular}{|c|c|c|c|}
\hline & LBBB IDCM $(n=26)$ & Non-LBBB IDCM $(n=75)$ & $P$ value \\
\hline Age (year) & $41.8 \pm 18.1$ & $35.6 \pm 18.7$ & 0.15 \\
\hline Male (\%) & $5(19.2 \%)$ & $42(56 \%)$ & 0.03 \\
\hline Female (\%) & $21(80.8 \%)$ & $33(44 \%)$ & \\
\hline BSA & $1.78 \pm 0.43$ & $1.69 \pm 0.52$ & 0.33 \\
\hline Familial (\%) & $1(4 \%)$ & $16(21 \%)$ & \\
\hline Symptoms & $2.04 \pm 0.73$ & $2.21 \pm 0.71$ & 0.34 \\
\hline \multicolumn{4}{|l|}{ Functional class (\%) } \\
\hline NYHA I & $7(21.2 \%)$ & $31(14.2 \%)$ & \multirow{4}{*}{0.16} \\
\hline NYHA II & $31(45.5 \%)$ & $9(64.3 \%)$ & \\
\hline NYHA III & $11(33.3 \%)$ & $2(14.3 \%)$ & \\
\hline NYHA IV & 0 & $1(7.1 \%)$ & \\
\hline HR (b/min) & $88.7 \pm 16.9$ & $90.9 \pm 19.5$ & 0.62 \\
\hline SBP (mmHg) & $108 \pm 19$ & $114 \pm 15$ & 0.18 \\
\hline $\mathrm{DBP}(\mathrm{mmHg})$ & $72 \pm 13$ & $73 \pm 11$ & 0.96 \\
\hline LA diameter $(\mathrm{mm})$ & $44.78 \pm 9.95$ & $44.96 \pm 11.15$ & 0.94 \\
\hline LA volume (ml) & $73.91 \pm 30.35$ & $76.18 \pm 46.61$ & 0.82 \\
\hline $\operatorname{ESD}(\mathrm{mm})$ & $60.46 \pm 12.88$ & $53.62 \pm 13.34$ & 0.03 \\
\hline EDD (mm) & $71.80 \pm 12.46$ & $64.48 \pm 13.40$ & 0.02 \\
\hline $\mathrm{EF} \%$ & $31.77 \pm 12.63$ & $35.09 \pm 13.16$ & 0.27 \\
\hline FS $\%$ & $15.85 \pm 6.79$ & $17.67 \pm 7.53$ & 0.28 \\
\hline Septum (mm) & $9.26 \pm 1.77$ & $8.96 \pm 2.53$ & 0.58 \\
\hline LVPW (mm) & $8.48 \pm 1.83$ & $8.31 \pm 2.34$ & 0.75 \\
\hline LVM (gm) & $364 \pm 149$ & $312 \pm 168$ & 0.17 \\
\hline $\operatorname{LVMI}\left(\mathrm{gm} / \mathrm{m}^{2}\right)$ & $207 \pm 78$ & $176 \pm 77$ & 0.08 \\
\hline \multicolumn{4}{|l|}{ Mitral regurge: } \\
\hline No & $13(50.0 \%)$ & $38(50.7 \%)$ & \multirow[t]{4}{*}{0.74} \\
\hline Mild & $0(0.0)$ & $1(1.3 \%)$ & \\
\hline Moderate & $6(23.1 \%)$ & $22(29.3 \%)$ & \\
\hline Severe & $7(26.9 \%)$ & $14(18.7 \%)$ & \\
\hline \multicolumn{4}{|l|}{ Tricuspid regurg: } \\
\hline No & $12(46.1)$ & $33(44 \%)$ & \multirow{4}{*}{0.92} \\
\hline Mild & $8(30.8)$ & $28(37.3)$ & \\
\hline Moderate & $4(15.4)$ & $10(13.3 \%)$ & \\
\hline Severe & $2(7.7)$ & $4(5.4 \%)$ & \\
\hline $\mathrm{E}(\mathrm{mm})$ & $74.48 \pm 30.67$ & $79.66 \pm 26.72$ & 0.44 \\
\hline$A(\mathrm{~mm})$ & $50.09 \pm 24.00$ & $50.52 \pm 21.09$ & 0.94 \\
\hline $\mathrm{E} / \mathrm{A}$ & $1.92 \pm 1.35$ & $1.98 \pm 1.32$ & 0.86 \\
\hline PAP (mmHg) & $39.6 \pm 11$ & $33.11 \pm 12$ & 0.03 \\
\hline DT (ms) & $150 \pm 62.5$ & $147.5 \pm 52.6$ & 0.85 \\
\hline $\mathrm{E}^{\prime}(\mathrm{cm} / \mathrm{s})$ & $8.34 \pm 3.9$ & $9.48 \pm 4.76$ & 0.32 \\
\hline $\mathrm{A}^{\prime}(\mathrm{cm} / \mathrm{s})$ & $74.5 \pm 30.7$ & $80.3 \pm 25.9$ & 0.38 \\
\hline $\mathrm{E} / \mathrm{E}^{\prime}$ & $11.61 \pm 8.73$ & $9.75 \pm 4.98$ & 0.23 \\
\hline
\end{tabular}

BSA: body surface area; DBP: systolic blood pressure; DBP: diastolic blood pressure. LAD: left atrium diameter; SPT: septal thickness, PWT: posterior wall thickness; EDD: left ventricular end diastolic diameter; ESD: left ventricular end systolic diameter; FS: fractional shortening; EF\% ejection fraction; LVMI: left ventricular mass index; PAP: pulmonary artery pressure; E: early diastolic mitral inflow velocity; $\mathrm{E}_{\mathrm{m}}$ : early diastolic myocardial velocity; $\mathrm{A}_{\mathrm{m}}$ : late diastolic myocardial velocity. 
Table 2. Left ventricular longitudinal strain $\left(\varepsilon_{\text {sys }}\right)$ and systolic strain rate in studied groups.

\begin{tabular}{|c|c|c|c|c|c|c|}
\hline & $\begin{array}{c}\text { IDCM } \\
\text { LBBB } \\
(n=26)\end{array}$ & $\begin{array}{c}\text { IDCM } \\
\text { Non LBBB } \\
(\mathrm{n}=75)\end{array}$ & $\begin{array}{c}P \\
\text { value }\end{array}$ & $\begin{array}{c}\text { IDCM } \\
\text { LBBB } \\
(\mathrm{N}=26)\end{array}$ & $\begin{array}{c}\text { IDCM } \\
\text { Non-LBBB } \\
(\mathrm{n}=75)\end{array}$ & $P$ value \\
\hline & $\varepsilon_{\text {sys } \%}$ & $\varepsilon_{\text {sys } \%}$ & & $\mathrm{SR}_{\text {sys }}\left(\mathrm{s}^{-1}\right)$ & $\mathrm{SR}_{\mathrm{sys}}\left(\mathrm{s}^{-1}\right)$ & \\
\hline \multicolumn{7}{|l|}{ Septum } \\
\hline Basal & $-3.38 \pm 3.11$ & $-5.34 \pm 4.26$ & 0.04 & $-0.39 \pm 0.18$ & $-0.49 \pm 0.27$ & 0.05 \\
\hline Mid & $-3.42 \pm 3.25$ & $-5.87 \pm 4.62$ & 0.02 & $-0.31 \pm 0.18$ & $-0.43 \pm 0.28$ & 0.02 \\
\hline Apical & $-2.55 \pm 2.73$ & $-5.31 \pm 4.42$ & 0.004 & $-0.28 \pm 0.19$ & $-0.36 \pm 0.27$ & 0.01 \\
\hline Mean & $-3.12 \pm 2.92$ & $-5.51 \pm 4.45$ & 0.005 & $-0.32 \pm 0.17$ & $-0.43 \pm 0.27$ & 0.02 \\
\hline \multicolumn{7}{|l|}{ Lateral } \\
\hline Basal & $-3.41 \pm 3.33$ & $-6.09 \pm 5.39$ & 0.02 & $-0.46 \pm 0.17$ & $-0.58 \pm 0.39$ & 0.11 \\
\hline Mid & $-2.61 \pm 2.78$ & $-4.95 \pm 4.43$ & 0.01 & $-0.33 \pm 0.14$ & $-0.48 \pm 0.42$ & 0.09 \\
\hline Apical & $-1.92 \pm 1.59$ & $-3.92 \pm 5.46$ & 0.07 & $-0.21 \pm 0.09$ & $-0.72 \pm 0.24$ & 0.42 \\
\hline Mean & $-2.45 \pm 3.34$ & $-4.26 \pm 5.09$ & 0.01 & $-0.33 \pm 0.13$ & $-0.59 \pm 0.32$ & 0.21 \\
\hline \multicolumn{7}{|l|}{ Anterior } \\
\hline Basal & $-4.53 \pm 4.58$ & $-6.66 \pm 5.34$ & 0.08 & $-0.46 \pm 0.23$ & $-0.57 \pm 0.39$ & 0.21 \\
\hline Mid & $-3.69 \pm 3.48$ & $-5.32 \pm 4.22$ & 0.09 & $-0.35 \pm 0.18$ & $-0.26 \pm 1.23$ & 0.70 \\
\hline Apical & $-2.59 \pm 1.69$ & $-3.34 \pm 2.92$ & 0.23 & $-0.24 \pm 0.09$ & $-0.49 \pm 2.02$ & 0.04 \\
\hline Mean & $-3.56 \pm 3.52$ & $-5.74 \pm 4.43$ & 0.13 & $-0.35 \pm 0.19$ & $-0.45 \pm 1.65$ & 0.32 \\
\hline \multicolumn{7}{|l|}{ Inferior } \\
\hline Basal & $3.69 \pm 2.31$ & $-7.29 \pm 6.83$ & 0.01 & $-0.52 \pm 0.27$ & $-0.59 \pm 0.48$ & 0.53 \\
\hline Mid & $-3.32 \pm 2.7$ & $-6.10 \pm 5.27$ & 0.01 & $-0.39 \pm 0.21$ & $-0.45 \pm 0.34$ & 0.35 \\
\hline Apical & $-3.01 \pm 2.1$ & $-4.26 \pm 3.5$ & 0.10 & $-0.25 \pm 0.14$ & $-0.69 \pm 0.34$ & 0.01 \\
\hline Mean & $-3.35 \pm 2.4$ & $-6.17 \pm 4.3$ & 0.02 & $-0.33 \pm 0.22$ & $-0.52 \pm 0.25$ & 0.06 \\
\hline Global & $-3.17 \pm 2.48$ & $-5.27 \pm 3.84$ & 0.01 & $-0.27 \pm 0.27$ & $-0.47 \pm 0.59$ & 0.03 \\
\hline
\end{tabular}

$\varepsilon_{\text {sys: }}$ : peak systolic strain; $\mathrm{SR}_{\text {sys }}$ : systolic strain rate.

Table 3. Left ventricular longitudinal early $\left(\mathrm{SR}_{\mathrm{e}}\right)$ and late $\left(\mathrm{SR}_{\mathrm{a}}\right)$ diastolic strain rate.

\begin{tabular}{ccccccc}
\hline & $\begin{array}{c}\text { IDCM } \\
\text { LBBB } \\
(\mathbf{n}=26)\end{array}$ & $\begin{array}{c}\text { IDCM } \\
\text { Non LBBB } \\
(\mathbf{n}=75)\end{array}$ & $\begin{array}{c}\mathbf{P} \\
\text { value }\end{array}$ & $\begin{array}{c}\text { IDCM } \\
\text { LBBB } \\
(\mathrm{N}=26)\end{array}$ & $\begin{array}{c}\text { IDCM } \\
\text { Non-LBBB } \\
(\mathbf{n}=75)\end{array}$ & P value \\
\hline & $\mathrm{SR}_{\mathrm{e}}\left(\mathrm{s}^{-1}\right)$ & $\mathrm{SR}_{\mathrm{e}}\left(\mathrm{s}^{-1}\right)$ & & $\mathrm{SR}_{\mathrm{a}}\left(\mathrm{s}^{-1}\right)$ & $\mathrm{SR}_{\mathrm{a}}\left(\mathrm{s}^{-1}\right)$ & \\
\hline Septum & & & & & & \\
Basal & $0.27 \pm 0.18$ & $0.41 \pm 0.36$ & 0.01 & $0.36 \pm 0.3$ & $0.37 \pm 0.3$ & 0.97 \\
Mid & $0.25 \pm 0.18$ & $0.39 \pm 0.31$ & 0.04 & $0.30 \pm 0.3$ & $0.32 \pm 0.2$ & 0.77 \\
Apical & $0.21 \pm 0.17$ & $0.34 \pm 0.27$ & 0.03 & $0.23 \pm 0.2$ & $0.26 \pm 0.3$ & 0.63 \\
Lateral & & & & & & \\
Basal & $0.41 \pm 0.36$ & $0.46 \pm 0.33$ & 0.46 & $0.29 \pm 0.2$ & $0.39 \pm 0.4$ & 0.19 \\
Mid & $0.33 \pm 0.29$ & $0.37 \pm 0.29$ & 0.59 & $0.21 \pm 0.1$ & $0.30 \pm 0.3$ & 0.10 \\
\hline
\end{tabular}




\begin{tabular}{ccccccc} 
Continued & \multicolumn{1}{c}{} & & & \\
\hline Apical & $0.19 \pm 0.14$ & $0.29 \pm 0.25$ & 0.06 & $0.10 \pm 0.1$ & $0.51 \pm 2.9$ & 0.48 \\
Anterior & & & & & & \\
Basal & $0.32 \pm 0.26$ & $0.55 \pm 0.48$ & 0.02 & $0.41 \pm 0.3$ & $0.37 \pm 0.3$ & 0.51 \\
Mid & $0.25 \pm 0.20$ & $0.41 \pm 0.35$ & 0.03 & $0.31 \pm 0.2$ & $0.29 \pm 0.2$ & 0.59 \\
Apical & $0.21 \pm 0.12$ & $0.25 \pm 0.19$ & 0.38 & $0.19 \pm 0.2$ & $0.16 \pm 0.1$ & 0.22 \\
Inferior & & & & & & \\
Basal & $0.49 \pm 0.47$ & $0.52 \pm 0.65$ & 0.90 & $0.38 \pm 0.2$ & $0.39 \pm 0.3$ & 0.83 \\
Mid & $0.34 \pm 0.31$ & $0.42 \pm 0.45$ & 0.42 & $0.36 \pm 0.2$ & $0.59 \pm 2.3$ & 0.61 \\
Apical & $0.21 \pm 0.15$ & $0.28 \pm 0.24$ & 0.21 & $0.17 \pm 0.1$ & $0.22 \pm 0.2$ & 0.19 \\
Global & $0.29 \pm 0.15$ & $0.39 \pm 0.25$ & 0.09 & $0.28 \pm 0.1$ & $0.34 \pm 0.4$ & 0.33 \\
\hline
\end{tabular}

$\mathrm{SR}_{\mathrm{e}}$ : early diastolic strain rate; $\mathrm{SR}_{\mathrm{a}}$ : atrial diastolic strain rate.

Table 4. LV mechanical dyssynchrony in studied groups.

\begin{tabular}{lccc}
\hline & $\begin{array}{c}\text { IDCM LBBB } \\
(\mathbf{n}=\mathbf{2 6})\end{array}$ & $\begin{array}{c}\text { IDCM non-LBBB } \\
(\mathbf{n}=\mathbf{7 5})\end{array}$ & P value \\
\hline TTP septum: Basal & $396.77 \pm 99$ & $340.52 \pm 116$ & 0.03 \\
$\quad$ Mid & $388.38 \pm 86$ & $343.06 \pm 123$ & 0.09 \\
$\quad$ Apical & $361.81 \pm 76$ & $324.89 \pm 125$ & 0.17 \\
TTP lateral: Basal & $409.73 \pm 102$ & $354.74 \pm 121$ & 0.04 \\
$\quad$ Mid & $400.54 \pm 78$ & $350.93 \pm 119$ & 0.06 \\
$\quad$ Apical & $361.85 \pm 77$ & $322.39 \pm 105$ & 0.09 \\
TTP anterior Basal & $386.9 \pm 80$ & $345.04 \pm 113$ & 0.09 \\
$\quad$ Mid & $383.1 \pm 71$ & $338.97 \pm 108$ & 0.06 \\
$\quad$ Apical & $365.8 \pm 84$ & $336.2 \pm 106$ & 0.21 \\
TTP inferior: Basal & $391.92 \pm 70$ & $355.53 \pm 121$. & 0.15 \\
$\quad$ Mid & $383.04 \pm 58$. & $365.51 \pm 109$ & 0.44 \\
$\quad$ Apical & $350.31 \pm 83$ & $332.10 \pm 103$ & 0.43 \\
TTP Mean & $444.45 \pm 92$ & $342.81 \pm 83$ & $<0.001$ \\
TTP min & $312.31 \pm 100$ & $236.28 \pm 92$ & 0.001 \\
TTP max & $586.81 \pm 127$. & $445.67 \pm 129$ & $<0.001$ \\
TTP-d & $274.5 \pm 116$ & $209.4 \pm 139$ & 0.02 \\
TTP-SD & $85.97 \pm 31.9$ & $67.10 \pm 40.7$ & 0.004 \\
Circum strain (\%) & $-3.44 \pm 5.83$ & $-3.14 \pm 4.39$ & 0.45 \\
Basal rotation $\left(^{\circ}\right)$ & $1.80 \pm 1.22$ & $1.53 \pm 1.30$ & 0.23 \\
Apical rotation $\left(^{\circ}\right)$ & $2.17 \pm 1.47$ & $2.25 \pm 1.83$ & 0.27 \\
LV twist $\left({ }^{\circ}\right)$ & $2.95 \pm 2.18$ & $2.97 \pm 2.22$ & 0.68 \\
\hline
\end{tabular}

TTP: time to peak systolic strain; TTP min: minimum TTP; TTP max: maximum TTP; TTP-SD: standard deviation of TTP.

non-LBBB group $(\mathrm{P}<0.02)$. This impaired strain rate values render $\mathrm{LV}$ global $\mathrm{SR}_{\mathrm{sys}}$ calculated from $12 \mathrm{LV}$ segments showed significant reduction in patients with $\mathrm{LBBB}\left(-0.27 \pm 0.27\right.$ versus $\left.-0.47 \pm 0.59 \mathrm{~s}^{-1}\right)$. Similarly, diastolic function represented by $\mathrm{SR}_{e}$ measured at mid $\mathrm{LV}$ septal segments were considerably lower in LBBB patients compared with non LBBB IDCM $(\mathrm{P}<0.03)$. 
Comparing the electromechanical delay between LV segments (TTP-d), it was greater in $\mathrm{LBBB}$ in comparison to non LBBB group $(274.5 \pm 116$ versus 209.4 $\pm 139, \mathrm{P}<0.02)$. The electromechanical delay between septal segments was $35 \pm$ $18 \mathrm{~ms}$ and between lateral wall segments: $48 \pm 24 \mathrm{~ms}$, between anterior wall segments: $21 \pm 11$ and between inferior wall segments: $41 \pm 12 \mathrm{~ms}$. Consequently, LV mechanical dyssynchrony was more evident in IDCM patients with LBBB compared to patients with no LBBB $(\mathrm{P}<0.004)$.

Regarding the circumferential strain and LV twist, there was no significant difference between LBBB group and non LBBB group.

\subsection{Relation of QRS Duration to LV Mechanics}

Univariate analysis was used to find the relation of QRS width, that reflects LV electrical activation, to all clinical and echocardiographic variables. Interestingly, IDCM patients with LBBB, QRS duration showed direct correlations only to SBP $(\mathrm{P}<0.04)$, EDD $(\mathrm{P}<0.04)$, E mitral inflow $(\mathrm{P}<0.04)$, and $\mathrm{E}^{\prime}(\mathrm{P}<0.04$. However in non $\mathrm{LBBB}$ group the $\mathrm{QRS}$ duration showed direct correlation to age $(\mathrm{P}<$ 0.001). BSA $(P<0.001)$, LVDd $(P=0.001)$ \& LVSd $(P=0.002)$, LVPW and LVM and PAP, A mitral inflow velocity, A' TDI on mitral annular. While LBBB IDCM group there was no relation of QRS width to $\mathrm{LV}$ mechanics $\left(\varepsilon_{\text {sys }} \%, \mathrm{SR}_{\text {sys }}\right)$. Non LBBB group showed inverse relationship to $\varepsilon_{\text {sys }} \%$ of septum $(\mathrm{P}=0.007)$, global LV $\varepsilon_{\text {sys }} \%(\mathrm{P}=0.005)$ (Figure 3$), \mathrm{TTP}-\mathrm{SD}(\mathrm{P}=0.02)$ (Figure 4$), \mathrm{SR}_{\text {sys }}(\mathrm{P}=0.04)$ (Figure 5) and $\operatorname{SRe}(\mathrm{P}=0.001)$ (Figure 6), Table 5.

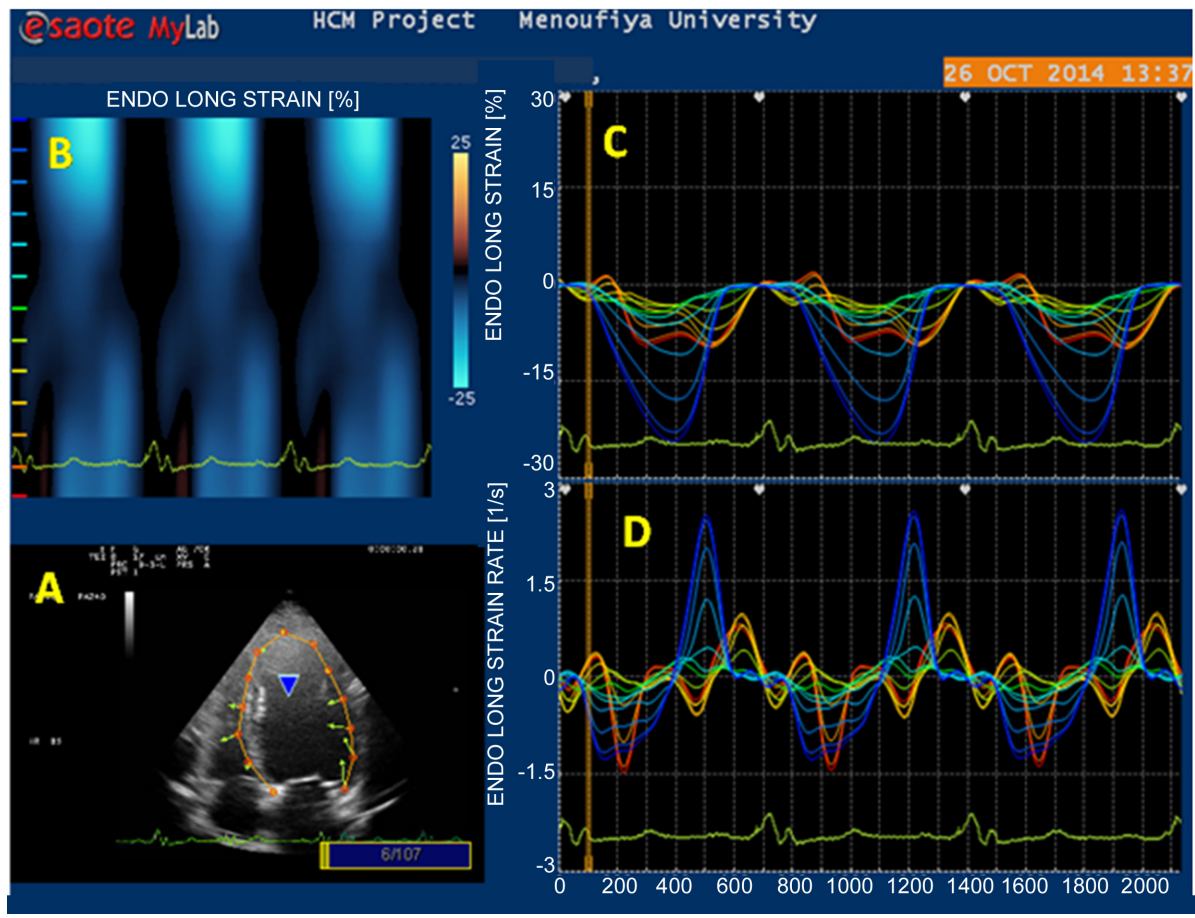

Figure 2. Strain and strain rate of LV segments in a patient with IDCM patient and LBBB A: LV tracking in apical $4 \mathrm{CH}$ view. B: Curved M-mode of septal and lateral wall segments. C: Strain curve of 6 segments derived from septal and lateral wall segments. D: Strain rate-time activity curve of same segments. 


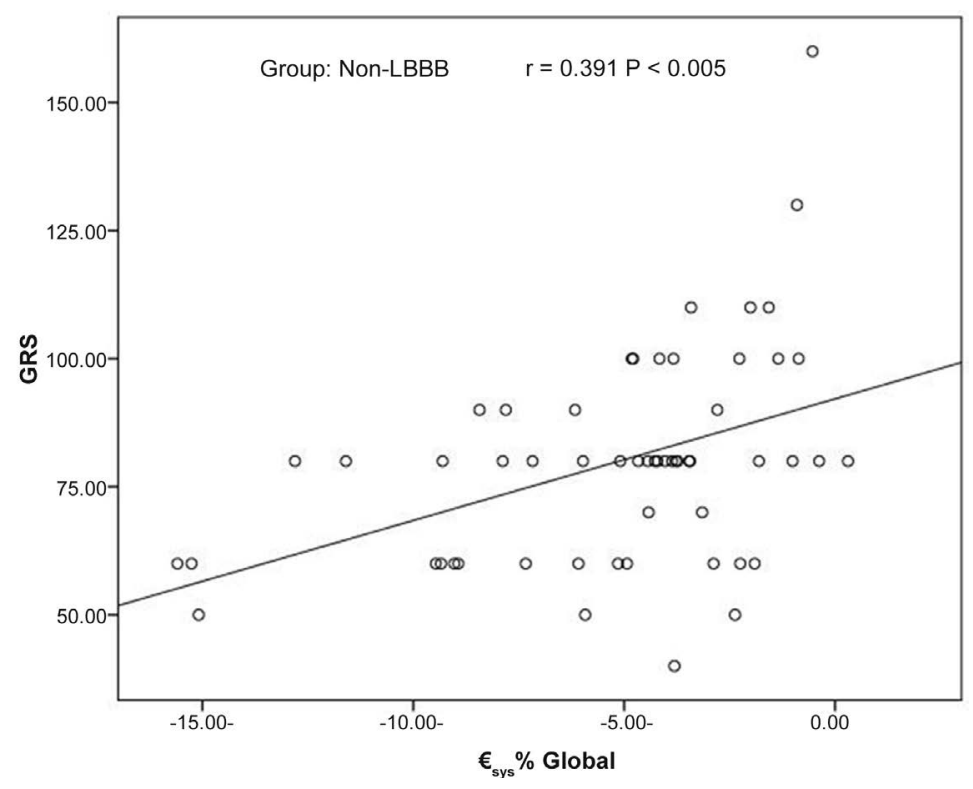

Figure 3. Correlation between QRS duration and global strain.

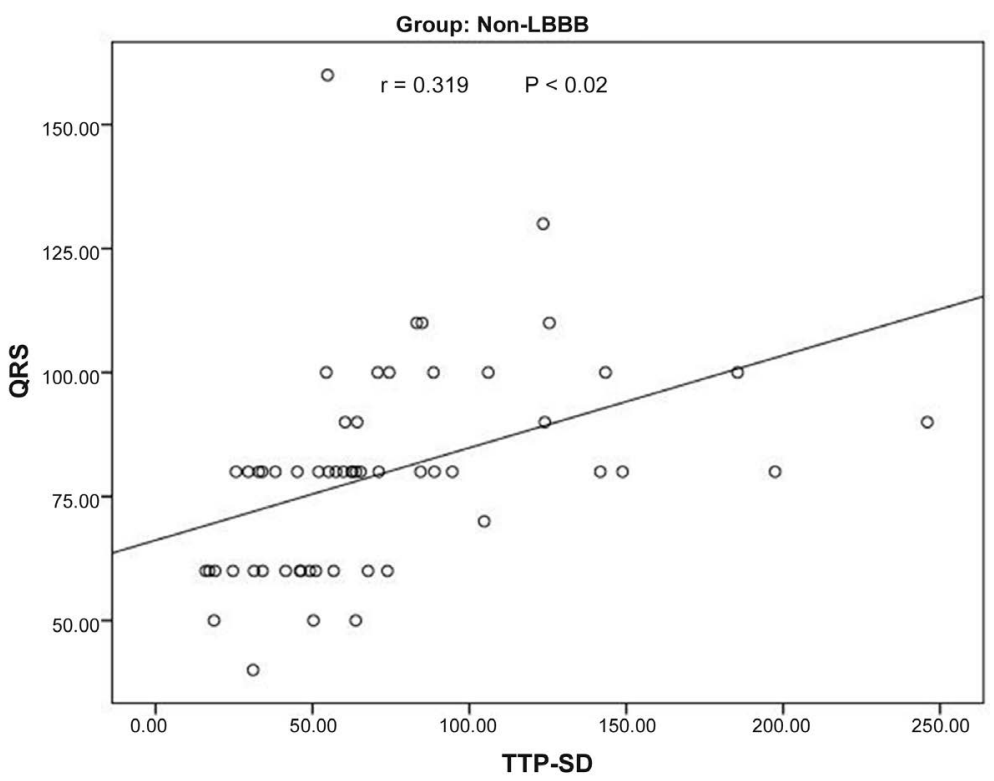

Figure 4. Relationship of QRS duration to SD of TTP (time to peak systolic strain).

Table 5. Relation of QRS duration and echocardiographic variables in studied groups.

\begin{tabular}{|c|c|c|c|c|c|c|c|c|c|}
\hline & \multicolumn{2}{|c|}{ IDCM LBBB } & \multicolumn{2}{|c|}{$\begin{array}{c}\text { IDCM } \\
\text { non-LBBB }\end{array}$} & & \multicolumn{2}{|c|}{ IDCM LBBB } & \multicolumn{2}{|c|}{$\begin{array}{c}\text { IDCM } \\
\text { non-LBBB }\end{array}$} \\
\hline & $\mathbf{r}$ & $\mathbf{P}$ & $\mathbf{r}$ & $\mathbf{P}$ & & $\mathbf{r}$ & $\mathbf{P}$ & $\mathbf{r}$ & $\mathbf{P}$ \\
\hline Age (y) & 0.162 & 0.43 & 0.430 & $<0.001$ & $\mathrm{E}(\mathrm{cm} / \mathrm{s})$ & 0.427 & $0.04^{*}$ & -0.168 & 0.22 \\
\hline BSA $\left(\mathrm{kg} / \mathrm{m}^{2}\right)$ & 0.242 & 0.23 & 0.423 & 0.001 & $\mathrm{~A}(\mathrm{~cm} / \mathrm{s})$ & 0.222 & 0.31 & -0.332 & 0.01 \\
\hline $\mathrm{HR}(\mathrm{b} / \mathrm{min})$ & -0.182 & 0.37 & 0.012 & 0.93 & $\mathrm{E} / \mathrm{A}$ & -0.071 & 0.75 & 0.309 & 0.02 \\
\hline SBP (mmHg) & 0.483 & $0.04^{*}$ & 0.032 & 0.83 & $\mathrm{DT}(\mathrm{ms})$ & -0.068 & 0.76 & -0.062 & 0.65 \\
\hline DBP (mmHg) & 0.324 & 0.21 & 0.027 & 0.86 & PAP (mmHg) & 0.323 & 0.17 & 0.452 & 0.001 \\
\hline LAD (mm) & 0.366 & 0.07 & 0.272 & 0.03 & $\mathrm{E}^{\prime}(\mathrm{cm} / \mathrm{s})$ & 0.427 & $0.04^{*}$ & -0.152 & 0.26 \\
\hline
\end{tabular}




\section{Continued}

\begin{tabular}{cccccccccc}
\hline LA V (ml) & -0.135 & 0.51 & 0.182 & 0.15 & $\mathrm{~A}^{\prime}(\mathrm{cm} / \mathrm{s})$ & -0.095 & 0.68 & -0.022 & 0.88 \\
LVSd (cm) & 0.210 & 0.30 & 0.382 & 0.002 & $\mathrm{E} / \mathrm{E}^{\prime}$ & 0.099 & 0.66 & -0.125 & 0.38 \\
LVDd (cm) & 0.397 & $0.04^{*}$ & 0.530 & 0.001 & $€_{\text {sys }} \%$ septum & 0.085 & 0.68 & 0.349 & 0.007 \\
FS\% & 0.167 & 0.42 & -0.129 & 0.31 & $€_{\text {sys }} \%$ Global & 0.192 & 0.35 & 0.391 & 0.005 \\
EF\% & 0.163 & 0.43 & -0.154 & 0.23 & TTP-d (ms) & -0.122 & 0.55 & 0.042 & 0.76 \\
LVPW (mm) & 0.225 & 0.27 & 0.398 & 0.001 & TTP-SD (ms) & -0.109 & 0.59 & 0.319 & 0.02 \\
LVM (gm) & 0.482 & $0.01^{*}$ & 0.469 & $<.001$ & TTP mean $(\mathrm{ms})$ & -0.089 & 0.67 & -0.092 & 0.50 \\
MR severity & -0.026 & 0.90 & 0.170 & 0.18 & SR $_{\text {sys }}\left(\mathrm{s}^{-1}\right)$ Global & -0.214 & 0.29 & 0.268 & 0.04 \\
TR severity & -0.251 & 0.22 & 0.202 & 0.11 & SR $_{e}$ Global $\left(\mathrm{s}^{-1}\right)$ & 0.196 & 0.34 & -0.444 & 0.001 \\
\hline
\end{tabular}

BSA: body surface area; DBP: diastolic blood pressure; DT: deceleration time; EF =: ejection fraction; FS: fraction shortening; LAD: left atrial diameter; LAV: left atrial volume; LVDd: left ventricular diastolic diameter; LVPW: left ventricular posterior wall; LVM: left ventricular mass; LVSd: left ventricular systolic diameter; MR: mitral regurgitation; PAP: pulmonary artery pressure; SBP: systolic blood pressure; TR: tricuspid regurgitation; TTP: time to peak systolic strain; $€_{\text {sys: }}$ systolic strain; $\mathrm{SR}_{\text {sys }}$ : systolic strain rate.

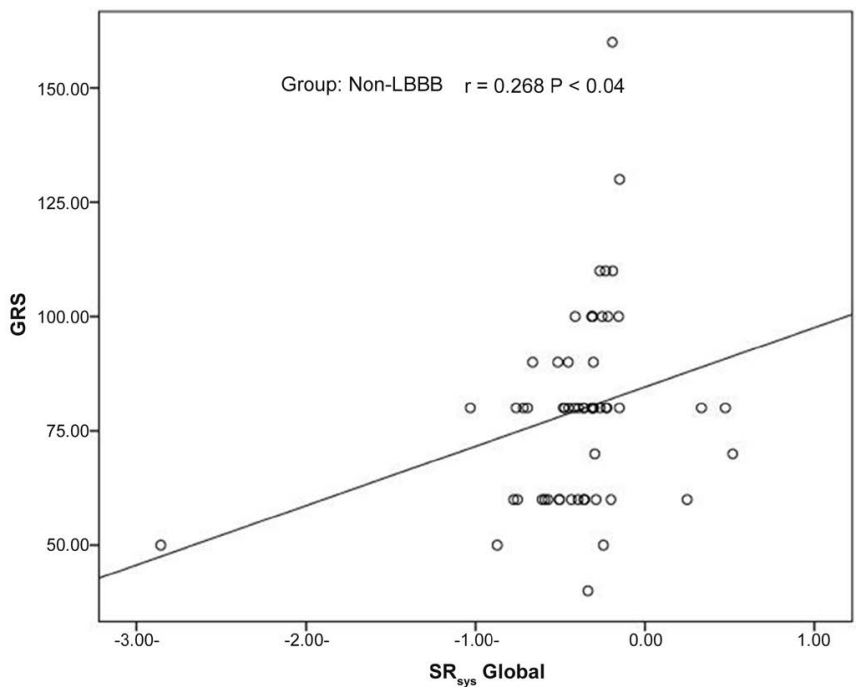

Figure 5. Relationship of QRS duration and global systolic strain rate $\left(\mathrm{SR}_{\mathrm{sys}}\right)$.

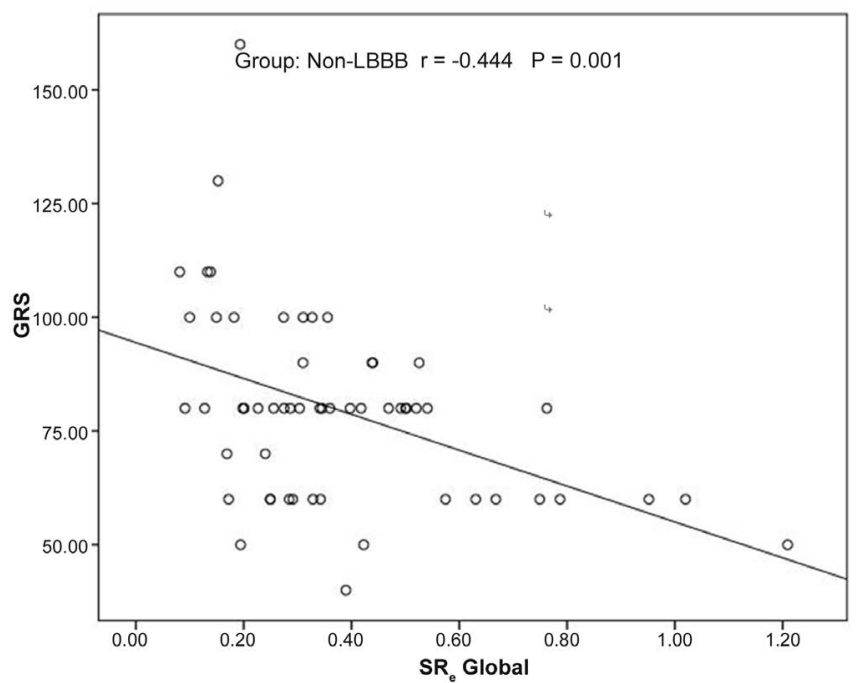

Figure 6. Relationship of $\mathrm{QRS}$ duration to early diastolic strain rate $\left(\mathrm{SR}_{\mathrm{e}}\right)$. 


\section{Discussion}

To our knowledge, this is the first study that provides an extensive evaluation of the influence of LBBB on LV mechanics in a single-centre, well characterized population of young patients with IDCM.

Dilated cardiomyopathy is an important cause of the heart failure. Advancements in cardiac imaging and introduction of speckle tracking are expected to provide better understanding of myocardial dysfunctions [12] [13].

From a mechanical perspective, patients with IDCM have an increased LV mass and volume due to the thinning and stretching of the LV wall [12]. These changes lead to reduced strain in all directions (e.g., longitudinal, radial, and circumferential) [13] [14] [15] [16]. As a result of these changes in the mechanics of the heart's pumping function, fewer symptoms are associated with higher cardiac strain. Similarly, dyssynchrony in circumferential and longitudinal strain predicts rapid HF progression. Likewise, LV rotation is reduced at the base and apex, which mitigates LV twisting and untwisting velocities [17]. The paradoxical reversal of LV rotation direction may be perceived with the LV base establishing counterclockwise rotation and the apex demonstrating clockwise rotation [18].

In the current study patients with IDCM showed marked reduction of strain values in all three directions, longitudinal, circumferential strain and deterioration of LV twist compared to values in healthy individuals in previous studies [12]-[18].

In our study, LBBB was present in approximately one-third of the IDCM patients at first presentation. This similar to ratio detected by Aleksova et al. [19] who were investigating 608 patients with DCM from the Heart Muscle Disease Registry of Trieste in retrospective analysis, they demonstrated that from study cohort, 189 patients (31.1\%) had LBBB.

Left bundle branch block is common among patients with HF, its prevalence depending on the severity of the HF [20].

Left bundle branch block has a negative impact on the cardiac performance. However, the published data exploring its impact on the outcome in patients with HF did not identify suitable explanations to this poor outcome [8] [9] [10] [12] [13] [14]. In a study by Tabrizi et al. [21], that included the patients with symptomatic HF requiring hospitalization, LBBB was not an independent predictor of mortality. Conversely, Baldasseroni et al. [22] found that the presence of LBBB increased the risk of 1 year all-cause mortality by $36 \%$ in a cohort of patients from the Italian Network CHF Registry. Brembilla-Perrot et al. [23] have analyzed this issue more recently in patients with DCM. However, they only included the patients with DCM in NYHAI-II, and reported higher event rates during follow-up in patients with LBBB or right bundle branch block. Grimm et al. [24] analyzed the predictors of survival of 343 patients with DCM. In their study were excluded patients in NYHA Class IV and LBBB has no impact on the subsequent outcome. 
In current study patients IDCM with LBBB had worse LV mechanics, they showed marked reduction of longitudinal strain of septal wall segments which is reflected by significant reduction of global LV longitudinal strain compared to non LBBB group, despite absence of difference in ejection fraction between both the two groups. Global LV longitudinal strain rate during systole and early diastole were consequently decreased in LBBB patients compared to non LBBB.

Additionally, LBBB showed marked deterioration of electromechanical activation and more LV dyssynchrony was observed in LBBB patient group.

\section{QRS Prolongation in IDCM}

Ventricular repolarization is a critical time in the cardiac cycle playing a considerable role in the pathophysiology of malignant arrhythmias. Its clinical evaluation should provide parameters that reflect cardiac electrical instability and, therefore, the increased risk of ventricular arrhythmias.

Despite several electrophysiological and pathological studies, the changes in ventricular repolarization in patients with IDCM remain unclear. In previous studies, prolongation of the QRS duration on an ECG served as an indirect marker to identify mechanical dyssynchrony [25].

The relation of QRS prolongation on the 12-lead ECG to LV dyssynchrony remains unclear and challenging. QRS duration is a simple measure of the duration of ventricular activation measured and is a manifestation of intraventricular or interventricular conduction delay.

Our study had several important implications. First, we demonstrated a reasonable explanation for poor outcome in HF patients with LBBB. While LV mechanical dysfunction was demonstrated in all IDCM patients, there was small proportion of them had LBBB with substantial deterioration of LV mechanics in patients with LBBB. Second LV mechanical dyssynchrony was similarly prevalent in both IDCM groups but with higher extent in IDCM with LBBB. Surprisingly, in LBBB patient group, LV deformation as measured by LV strain and strain rate was not related to QRS width which is the main criteria for LBBB diagnosis. However LV mechanics and dyssynchrony were related to QRS width in IDCM without LBBB. This could be due to the fact that the QRS width as an ECG parameters, poorly reflect the complexity of the ventricular mechanics and the heterogonous contraction present in $\mathrm{LBBB}$, but could be a valuable sign for mechanical function deterioration in non LBBB deteriorarion before LBBB become manifest.

In this study, QRS prolongation ( $>120 \mathrm{~ms}$ ) on 12 lead ECG was detected in $20 \%$ of IDC patients; $\sim 63 \%$ of them had LBBB. This prevalence is in accordance to previous studies that reported QRS prolongation in approximately $30 \%$ of adult patients with advanced heart failure [19] and left bundle branch block was predominating [26].

In our study in LBBB group: QRS duration was associated with increased LV dimension, LV mass and LA volume but not related to mechanical dysfunction 
or dyssynchrony. Chen et al. [27] explored the role of QRS prolongation ( $>120$ $\mathrm{ms}$ ) and its relation to mechanical dyssynchrony and outcomes in 89 patients $\leq$ 18 years old using tissue Doppler imaging. While they reported that longer QRS duration tended to increase the risk of requiring intravenous inotropes at follow-up, and strongly predicted poor clinical outcomes; there was poor correlation between $\mathrm{QRS}$ duration and intra- $\mathrm{V}$ dyssynchrony. They suggested a limitation of using QRS duration to predict mechanical intra-LV dyssynchrony.

A report by Friedberg et al. [16] examined the association between mechanical dyssynchrony and clinical status in 32 children with IDC. They indicated that intra-LV dyssynchrony was prevalent in $65 \%$ of patients with IDC. However, there was no difference in QRS duration between synchronous and non synchronous patients. Also, QRS duration alone was inadequate to define dyssynchrony in IDC patients and the role played by QRS prolongation was not clearly defined.

Moreover, Bleeker et al. [28] examined the relation of QRS duration to LV mechanical dyssynchrony using TDI in ninety patients with severe heart failure (left ventricular ejection fraction $<35 \%$ ). Severe dyssynchrony was observed in $70 \%$ of patients with wide QRS complex (>150 ms). However, no relation existed between QRS duration and septal-to-lateral delay. Furthermore, a growing experience with CRT in adults has highlighted the limitation of using QRS prolongation only to predict mechanical dyssynchrony.

\section{Study Limitations}

Data on mechanical dysfunction and dyssynchrony were obtained from a relatively small portion of patients with IDCM; therefore, profiles of mechanical dyssynchrony demonstrated in this study may not be exactly the same as those in the entire population with IDCM.

In our study no follow up study was performed on patients populations. The conduction disturbances are associated with more advanced stages of mechanical dysfunction. The development of LBBB and its specific role in long-term prognosis may thus be difficult to be interpreted. The expected poor outcome of patients with LBBB and advanced mechanical dysfunction come when considered as a time-dependent variable, and after adjustment for all other clinical prognostic baseline variables.

\section{Conclusion}

LBBB is frequent among the patients with IDCM; it adversely affects cardiac mechanics especially in longitudinal direction. Mechanical dyssynchrony is common association of electrical dyssynchrony in IDCM patients when LBBB exists. QRS duration prolongation might be a preceding stage, before LBBB develops. It is correlated with mechanical dysfunction in IDCM without LBBB. Deterioration of longitudinal strain and mechanical dyssynchrony in IDCM are reasonable explanation of poor outcome in IDCM patients with LBBB. Further 
studies may be warranted to prove this relationship.

\section{Conflicts of Interest}

The authors declare no conflicts of interest regarding the publication of this paper.

\section{References}

[1] Lindner, O., Vogt, J., Baller, D., Kammeier, A., Wielepp, P., Holzinger, J., Lamp, B., Horstkotte, D. and Burchert, W. (2005) Global and Regional Myocardial Oxygen Consumption and Blood Flow in Severe Cardiomyopathy WITH Left Bundle Branch Block. The European Journal of Heart Failure, 7, 225-230. https://doi.org/10.1016/j.ejheart.2004.07.003

[2] Masci, P.G., Marinelli, M., Piacenti, M., Lorenzoni, V., Positano, V., Lombardi, M., L'Abbate, A. and Neglia, D. (2010) Myocardial Structural, Perfusion, and Metabolic Correlates of Left Bundle Branch Block Mechanical Derangement in Patients With Dilated Cardiomyopathy: A Tagged Cardiac Magnetic Resonance and Positron Emission Tomography Study. Circulation: Cardiovascular Imaging, 3, 482-490. https://doi.org/10.1161/CIRCIMAGING.109.934638

[3] Blanc, J.J., Fatemi, M., Bertault, V., Baraket, F. and Etienne, Y. (2005) Evaluation of Left Bundle Branch Block as a Reversible Cause of Non-Ischaemic Dilated Cardiomyopathy with Severe Heart Failure. A New Concept of Left Ventricular Dyssynchrony-Induced Cardiomyopathy. Europace, 7, 604-610. https://doi.org/10.1016/j.eupc.2005.06.005

[4] Littmann, L. and Symanski, J.D. (2000) Hemodynamic Implications of Left Bundle Branch Block. Journal of Electrocardiology, 33, 115-121.

https://doi.org/10.1054/jelc.2000.20330

[5] Abduch, M.C., Salgo, I., Tsang, W., Vieira, M.L., Cruz, V., Lima, M., Tsutsui, J.M., Mor-Avi, V., Lang, R.M. and Mathias Jr., W. (2012) Myocardial Deformation by Speckle Tracking in Severe Dilated Cardiomyopathy. Arquivos Brasileiros de Cardiologia, 99, 834-843. https://doi.org/10.1590/S0066-782X2012005000086

[6] Richardson, P., McKenna, W., Bristow, M., et al. (1996) Report of the 1995 World Health Organization/International Society and Federation of Cardiology Task Force on the Definition and Classification of Cardiomyopathies. Circulation, 93, 841-842. https://doi.org/10.1161/01.CIR.93.5.841

[7] Daubeney, P.E., Nugent, A.W., Chondros, P., Carlin, J.B., Colan, S.D., Cheung, M., Davis, A.M., Chow, C.W. and Weintraub, R.G. (2006) Clinical Features and Outcomes of Childhood Dilated Cardiomyopathy: Results from a National Population-Based Study. Circulation, 114, 2671-2678. https://doi.org/10.1161/CIRCULATIONAHA.106.635128

[8] Badran, H.M., Elnoamany, M.F. and Seteha, M. (2007) Tissue Velocity Imaging with Dobutamine Stress Echocardiography-A Quantitative Technique for Identification of Coronary Artery Disease in Patients with Left Bundle Branch Block. Journal of the American Society of Echocardiography, 20, 820-831. https://doi.org/10.1016/j.echo.2007.01.007

[9] Chou, T. (1996) Electrocardiography in Clinical Practice: Adult and Pediatric. WB Saunders, Philadelphia, 37-120.

[10] Yi, G., Elliott, P., McKenna, W.J., Prasad, K., Sharma, S., Guo, X.H., et al. (1998) QT dispersion and Risk Factors for Sudden Cardiac Death in Patients with Hyper- 
trophic Cardiomyopathy. American Journal of Cardiology, 82, 1514-1519. https://doi.org/10.1016/S0002-9149(98)00696-1

[11] Maron, B.J., McKenna, W.J., Danielson, G.K., Kappenberger, L.J., Kuhn, H.J., Seidman, C.E., et al. (2003) American College of Cardiology/European Society of Cardiology Clinical Expert Consensus Document on Hypertrophic Cardiomyopathy. A report of the American College of Cardiology Foundation Task Force on Clinical Expert Consensus Documents and the European Society of Cardiology Committee for Practice Guidelines. European Heart Journal, 24, 1965-1991. https://doi.org/10.1016/S0195-668X(03)00479-2

[12] Wu, Y.T., Chang, A.C. and Chin, A.J. (1993) Semiquantitative Assessment of Mitral Regurgitation by Doppler Color Flow Imaging in Patients Aged > 20 Years. American Journal of Cardiology, 71, 727-732. https://doi.org/10.1016/0002-9149(93)91018-D

[13] Vannan, M.A., Pedrizzetti, G., Li, P., Gurudevan, S., Houle, H., Main, J., et al. (2005) Effect of Cardiac Resynchronization Therapy on Longitudinal and Circumferential Leftventricular Mechanics by Velocity Vector Imaging: Description and Initial Clinical Application of a Novel Method Using High-Frame Rate B-Mode Echocardiographic Images. Echocardiography, 22, 826-830. https://doi.org/10.1111/j.1540-8175.2005.00172.x

[14] Takamura, T., Dohi, K., Onishi, K., et al. (2010) Left Ventricular Contraction-Relaxation Coupling in Normal, Hypertrophic, and Failing Myocardium Quantified by Speckle-Tracking Global Strain and Strain Rate Imaging. Journal of the American Society of Echocardiography, 23, 747-754. https://doi.org/10.1016/j.echo.2010.04.005

[15] Zeng, S., Zhou, Q.C., Peng, Q.H., et al. (2009) Assessment of Regional Myocardial Function in Patients with Dilated Cardiomyopathy by Velocity Vector Imaging. Echocardiography, 26, 163-170. https://doi.org/10.1111/j.1540-8175.2008.00797.x

[16] Friedberg, M.K. and Slorach, C. (2008) Relation between Left Ventricular Regional Radial Function and Radial Wall Motion Abnormalities Using Two-Dimensional Speckle Tracking in Children with Idiopathic Dilated Cardiomyopathy. American Journal of Cardiology, 102, 335-339. https://doi.org/10.1016/j.amjcard.2008.03.064

[17] Jasaityte, R., Dandel, M., Lehmkuhl, H., et al. (2009) Prediction of Short-Term Outcomes in Patients with Idiopathic Dilated Cardiomyopathy Referred for Transplantation Using Standard Echocardiography and Strain Imaging. Transplantation Proceedings, 41, 277-280. https://doi.org/10.1016/j.transproceed.2008.10.083

[18] Meluzin, J., Spinarova, L., Hude, P., et al. (2009) Left Ventricular Mechanics in Idiopathic Dilated Cardiomyopathy: Systolic-Diastolic Coupling and Torsion. Journal of the American Society of Echocardiography, 22, 486-493. https://doi.org/10.1016/j.echo.2009.02.022

[19] Aleksova, A., Carriere, C., Zecchin, M., Barbati, G., Vitrella, G., Di Lenarda, A. and Sinagra, G. (2014) New-Onset Left Bundle Branch Block Independently Predicts Long-Term Mortality in Patients with Idiopathic Dilated Cardiomyopathy: Data from the Trieste Heart Muscle Disease Registry. Europace, 16, 1450-1459. https://doi.org/10.1093/europace/euu016

[20] Popovic, Z.B., Grimm, R.A., Ahmad, A., et al. (2008) Longitudinal Rotation: An Unrecognised Motion Pattern in Patients with Dilated Cardiomyopathy. Heart, 94, 11. https://doi.org/10.1136/hrt.2007.122192

[21] Tabrizi, F., Englund, A., Rosenqvist, M., Wallentin, L. and Stenestrand, U. (2007) Influence of Left Bundle Branch Block on Long-Term Mortality in a Population 
with Heart Failure. European Heart Journal, 28, 2449-2455.

https://doi.org/10.1093/eurheartj/ehm262

[22] Baldasseroni, S., Gentile, A., Gorini, M., Marchionni, N., Marini, M., Masotti, G., et al. (2003) Italian Network on Congestive Heart Failure Investigators. Intraventricular Conduction Defects in Patients with Congestive Heart Failure: Left But Not Right Bundle Branch Block Is an Independent Predictor of Prognosis. A Report from the Italian Network on Congestive Heart Failure (IN-CHF Database). Italian Heart Journal, 4, 607-613. https://doi.org/10.1016/S0195-668X(03)95854-4

[23] Brembilla-Perrot, B., Alla, F., Suty-Selton, C., Huttin, O., Blangy, H., Sadoul, N., Chometon, F., Groben, L., Luporsi, J.D., Zannad, N., Aliot, E., Cedano, J., Ammar, S., Abdelaal, A. and Juillière, Y. (2008) Nonischemic Dilated Cardiomyopathy: Results of Noninvasive and Invasive Evaluation in 310 Patients and Clinical Significance of Bundle Branch Block. Pacing and Clinical Electrophysiology, 31, 1383-1390. https://doi.org/10.1111/j.1540-8159.2008.01199.x

[24] Grimm, R.A., De, S., Popović, Z.B., Verhaert, D., Dresing, T., Wilkoff, B., Starling, R., Tang, W.H. and Thomas, J.D. (2015) Comparison of Left Ventricular Torsion and Strain with Biventricular Pacing in Patients with Underlying Right Bundle Branch Block versus Those with Left Bundle Branch Block. American Journal of Cardiology, 115, 918-923. https://doi.org/10.1016/j.amjcard.2015.01.018

[25] Shenkman, H.J., Pampati, V., Khandelwal, A.K. and McKinnon, J. (2002) Congestive Heart Failure and QRS Duration: Establishing Prognosis Study. Chest, 122, 528-534. https://doi.org/10.1378/chest.122.2.528

[26] Li, P., Tonti, G., Verjans, J., Pedrizzetti, G., Metha, H. and Appleby, S. (2005) Measurement of Apical Torsion in Mitochondrial Cardiomyopathy Using a Novel B-Mode, Automated Tracking Algorithm. Journal of the American College of Cardiology, 45, 305A.

[27] Chen, C.A., Hsiao, C.H., Wang, J.K., Lin, M.T., Wu, E.T., Chiu, S.N., Chiu, H.H. and $\mathrm{Wu}, \mathrm{M} . \mathrm{H}$. (2009) Implication of QRS Prolongation and Its Relation to Mechanical Dyssynchrony in Idiopathic Dilated Cardiomyopathy in Childhood. American Journal of Cardiology, 103, 103-109. https://doi.org/10.1016/j.amjcard.2008.08.044

[28] Bleeker, G.B., Mollema, S.A., van der Wall, E.E., Schalij, M.J. and Bax, J.J. (2007) Usefulness of QRS Duration to Predict Response to Cardiac Resynchronization Therapy in Patients with End-Stage Heart Failure. American Journal of Cardiology, 100, 1665-1670. https://doi.org/10.1016/j.amjcard.2007.06.071 